\title{
Suitability of Lock-in Infrared Thermography for Luminescent Glass Development
}

\author{
by Peter W. Nolte**, Nils Ziegeler*, A. Charlotte Rimbach*, Timo Malvisalo*, Stefan Schweizer ${ }^{* * *}$ \\ * South Westphalia University of Applied Sciences, Faculty of Electrical Engineering, Lübecker Ring 2, \\ 59494 Soest, Germany \\ ** Fraunhofer Application Center for Inorganic Phosphors, Branch Lab of Fraunhofer Institute for Microstructure \\ of Materials and Systems IMWS, Lübecker Ring 2, 59494 Soest, Germany, peter.nolte@imws.fraunhofer.de
}

\begin{abstract}
The purpose of this work is to evaluate the suitability of lock-in infrared thermography for luminescent glass development. Lithium borate glass of different composition is fabricated and studied to detect changes in the thermal diffusivity as the composition changes and property modifiers are introduced. In addition, the effect of terbium doping on the thermal diffusivity of the glass is studied.
\end{abstract}

\section{Introduction}

In white-light emitting diode (W-LED) applications, luminescent borate glass represents an attractive alternative to the commonly used phosphor-polymer-composite due to their better thermal and chemical stability [1-3]. Knowledge of the thermal properties of the used glass is important, since the temperature of the LED packaging affects the lifetime of the components as well as the efficiency of the LED and the apparent color of the light emitted [4-6]. This work describes the potential of lock-in infrared thermography to determine the thermal diffusivity of these glasses.

As infrared detection systems are getting more and more advanced, measurements can be performed on a wider range of materials with increasing accuracy. In combination with the advantages of lock-in techniques, reliable results can be achieved with low effort and high accuracy. This makes the usage of infrared imaging attractive for the analysis of thermal parameters, such as the thermal diffusivity and the thermal conductivity.

Exact knowledge of the emissivity is a common problem when dealing with infrared imaging. An advantage of the presented method is that the actual emissivity of the sample's surface is not important, since the absolute temperature of the sample is of no interest. Equally negligible are all time independent effects, such as reflections or surface structure inhomogeneities of the material, which alter the infrared radiation. In addition, averaging over several duty cycles allows a sufficient signal-noise ratio to detect weak signals with high sensitivity.

In this work, laser-based lock-in infrared measurements are conducted to determine the thermal diffusivities of various glasses. As opposed to other methods, which require direct contact of a heater source and a thermocouple to the sample, this method achieves good reproducibility and is less prone to errors. Here, pulsed laser excitation is used for periodically heating of the glass surface. The phase delay of the heat wave traveling through the sample is then recorded as a function of the lock-in frequency. For each sample, the lock-in frequency is varied within a specific range and the corresponding phase delay is subsequently plotted versus the lock-in frequency. To obtain the thermal diffusivity, a curve fitting of the obtained phase values is carried out. The resultant fitting parameter is then used to calculate the thermal diffusivity.

\section{Theoretical Background}

\subsection{Thermal Waves}

Measuring the thermal diffusivity with lock-in infrared thermography relies on the knowledge of the nature of propagation of thermal waves in the medium. A thermal wave is an oscillation of the temperature field in time and space. Since heat conduction is a diffusive phenomenon by nature, a thermal wave can only exist if it is driven by an external source. Hence, thermal waves are missing key concepts comprised in the classical wave concept, such as propagation speed and energy transport.

In the experiment, the external source is realised by the absorption of a pulsed laser beam. Since the laser can only ever increase the energy of the sample, the temperature gains and losses of the oscillations are formulated with reference to a thermal equilibrium. Its actual value depends on the average energy flux into the sample and its thermal contact to the environment. In lock-in thermography, only the alternating part of the signal is of interest; the direct part is disregarded.

The thermal modelling of a heatwave traveling though the sample is crucial for the analysis of the thermal diffusivity. The heat equation (Eq. (1)) should be solved under appropriate boundary conditions, initial values, and source distributions: 


$$
\nabla^{2} T(x, t)-\frac{1}{\alpha} \frac{\partial T(x, t)}{\partial t}=-\frac{\rho(x, t)}{k}
$$

with $T(x, t)$ the temperature distribution, $\alpha$ the thermal diffusivity, $k$ the thermal conductivity, and $\rho(x, t)$ the volumetric heat source. This has been applied for many different geometries and heating patterns. In the following, a semi-infinite half space heated by a point like source that oscillates harmonically with the frequency $\omega$ on the surface is considered. The solution becomes a one dimensional spherical thermal wave with amplitude $A$, which can be expressed as a function of the radius $r$ [7],

$$
T(r, t)=\frac{A}{r} \cdot e^{-r / \mu} \cdot e^{\mathrm{i}(\omega t-r / \mu)}
$$

Here, the thermal diffusion length

$$
\mu=\sqrt{2 \alpha / \omega}
$$

is introduced characterising the typical length scale of the problem.

In practice, the sample has a finite thickness. To evaluate whether the spherical thermal wave is still a good approximation for the real temperature field, the sample thickness, $d$, must be compared to the thermal diffusion length, $\mu$. As long as $\mu<<d$ holds, the spherical thermal wave is a good approximation to the real temperature field. As $\mu$ is a function of $\omega$, there result some consequences for the lock-in frequency, as it will be discussed later.

Above, a harmonically oscillating point like source has been assumed. As it is difficult to realise an ideal harmonic oscillation in practice, it is of interest to consider the influence of deviations from harmonic behaviour. As long as the heat influx is periodic in time, the incoming signal $\rho(t)$ comprises its base frequency and higher harmonics,

$$
\rho(r, t)=\frac{\rho_{0}}{2}+\rho_{0}\left(\frac{2}{\pi} \sin (\omega t)+\frac{2}{3 \pi} \sin (3 \omega t)+\ldots\right)
$$

If the thermal diffusivity, $\alpha$, and the thermal conductivity, $k$, show no temperature dependence, the heat equation is linear and thus the higher harmonics superimpose linearly. But, since the thermal diffusion length is decreasing with increasing frequency (compare Eq. (3)), the higher harmonics show a stronger damping. Thus, the thermal wave becomes more and more sinusoidal as is travels though the sample. If the criterion $\mu<d$ is fulfilled, the measured signal on the opposite side can be considered as sinusoidal.

\subsection{Data Analysis}

As it can be seen from Eq. (2), the temperature oscillation on the backside of the sample has a constant phase offset, $\varphi(\mu)=r / \mu$, which depends on the thermal diffusion length, $\mu$. If the phase delay is measured directly opposite of the heat influx, the distance $r$ equals the sample's thickness, $d$. This defines a proportionality constant, $b$, between the phase and the square root of the lock-in frequency, which can be solved for the thermal diffusivity, $\alpha$ :,

$$
\varphi=-d \sqrt{\frac{\pi}{\alpha}} \sqrt{f}+\varphi_{0}=b \sqrt{f}+\varphi_{0} .
$$

Here, a constant offset, $\varphi_{0}$, is introduced to account for a potential phase delay from the measurement system. Normalizing the phase values to the sample's thickness leads to

$$
\frac{\varphi-\varphi_{0}}{d}=-\sqrt{\frac{\pi}{\alpha}} \sqrt{f}=b_{\text {norm }} \sqrt{f}
$$

A series of measurements with different frequencies allows the analysis of the parameter $b_{\text {norm, }}$ which represents the slope of a thickness normalized phase versus square root of the lock-in frequency graph. 


\section{Experimental Details}

\subsection{Samples}

The glass samples investigated comprise four different sample sets. For all samples, the base components are boron oxide $\left(\mathrm{B}_{2} \mathrm{O}_{3}\right)$ as network former and lithium oxide $\left(\mathrm{Li}_{2} \mathrm{O}\right)$ as network modifier. Series with different modifier-toformer ratio are made ( $\mathrm{LiB}$ sample set). The property modifier aluminum oxide $\left(\mathrm{Al}_{2} \mathrm{O}_{3}\right)$ is added for higher chemical stability and to reduce hygroscopicity (LiAIB sample set). Here, the aluminum oxide is added at the expense of boron oxide, i.e., $10 \mathrm{~mol} \%$ of the boron oxide is substituted by aluminum oxide. The exact compositions are listed in table 1.

Furthermore, the above described sample sets are doped with different amounts of terbium oxide $\left(\mathrm{Tb}_{4} \mathrm{O}_{7}\right)$ for optical activation ( $\mathrm{LiB}: \mathrm{Tb}$ and LiAIB:Tb sample sets). For the optically activated sample sets, a $\mathrm{Li}_{2} \mathrm{O}$ concentration of $25 \mathrm{~mol} \%$ is used. The glass components are mixed and melted in a platinum gold crucible (Pt/Au 95/5) at $1000{ }^{\circ} \mathrm{C}$ for approximately $3 \mathrm{~h}$. For further details on the glass preparation, the reader is referred to $[8,9]$.

To guarantee a sufficient absorption of the laser pulses and to enable an efficient emission of the infrared radiation, the glasses are painted black on both sides. The paint is applied as thin as possible to minimize its influence on the thermal properties of the glasses. The thickness of the sample, $d$, is measured with a micrometer at the location where the laser pulses hit the sample.

Table 1. Nominal composition, terbium content, and thickness of the investigated lithium borate glass samples.

\begin{tabular}{|c|c|c|c|c|c|c|}
\hline sample set & \multicolumn{4}{|c|}{ composition / mol\% } & $\begin{array}{l}\mathrm{Tb}^{3+} \text { content } \\
\text { l at. } \%\end{array}$ & $\begin{array}{l}\text { thickness } \\
\text { / mm }\end{array}$ \\
\hline \multirow[t]{4}{*}{ LiB } & 85.71 & 14.29 & - & - & - & $1.36 \pm 0.05$ \\
\hline & 83.33 & 16.67 & - & - & - & $1.05 \pm 0.05$ \\
\hline & 80.00 & 20.00 & - & - & - & $1.17 \pm 0.05$ \\
\hline & 75.00 & 25.00 & - & - & - & $1.29 \pm 0.05$ \\
\hline \multirow[t]{5}{*}{ LiAIB } & 77.14 & 14.29 & 8.57 & - & - & $1.35 \pm 0.05$ \\
\hline & 75.00 & 16.67 & 8.33 & - & - & $1.21 \pm 0.05$ \\
\hline & 72.00 & 20.00 & 8.00 & - & - & $1.22 \pm 0.05$ \\
\hline & 67.50 & 25.00 & 7.50 & - & - & $1.32 \pm 0.05$ \\
\hline & 60.00 & 33.33 & 6.67 & - & - & $1.16 \pm 0.05$ \\
\hline \multirow[t]{3}{*}{$\mathrm{LiB}: \mathrm{Tb}$} & 72.80 & 24.27 & - & 2.93 & 2.5 & $1.40 \pm 0.05$ \\
\hline & 73.70 & 24.57 & - & 1.73 & 1.5 & $1.05 \pm 0.05$ \\
\hline & 74.57 & 24.86 & - & 0.57 & 0.5 & $1.17 \pm 0.05$ \\
\hline \multirow[t]{3}{*}{ LiAIB:Tb } & 65.52 & 24.27 & 7.28 & 2.93 & 2.5 & $1.32 \pm 0.05$ \\
\hline & 66.33 & 24.57 & 7.37 & 1.73 & 1.5 & $1.32 \pm 0.05$ \\
\hline & 67.11 & 24.86 & 7.46 & 0.57 & 0.5 & $1.32 \pm 0.05$ \\
\hline
\end{tabular}

\subsection{Experimental Setup}

The experiments are performed with a lock-in thermography setup consisting of an infrared camera (InfraTec ImageIR 8380S) and a 980-nm laser diode (Thorlabs L980P100) with an (optical) output power of $100 \mathrm{~mW}$ for periodic heating. The IR camera uses an indium antimonide (InSb) focal plane array (FPA) snapshot detector with a geometric resolution of $640 \mathrm{px} \times 512 \mathrm{px}$; the spectral range for detection is between $2.0 \mu \mathrm{m}$ and $5.7 \mu \mathrm{m}$. For lock-in measurements, IR camera and laser are connected via a control box synchronizing the image capturing and the laser pulses for different lock-in frequencies. The laser is switched on and off by the synchronization box.

The samples are placed into an evacuated vacuum chamber with a pressure below $0.2 \mathrm{mbar}$ to exclude heat losses through convection and to guarantee reproducible boundary conditions. The laser beam excitation occurs through a borosilicate glass, while the IR camera records the infrared radiation through a sapphire glass. The sapphire glass window provides a transmission of $90 \%$ in the spectral range between $2 \mu \mathrm{m}$ and $4 \mu \mathrm{m}$, while in the range between $4 \mu \mathrm{m}$ and $5.7 \mu \mathrm{m}$ the transmission decreases almost linearly to $40 \%$. The borosilicate glass is transparent for the used laser wavelength of $980 \mathrm{~nm}$. The experimental setup is depicted in figure 1. 


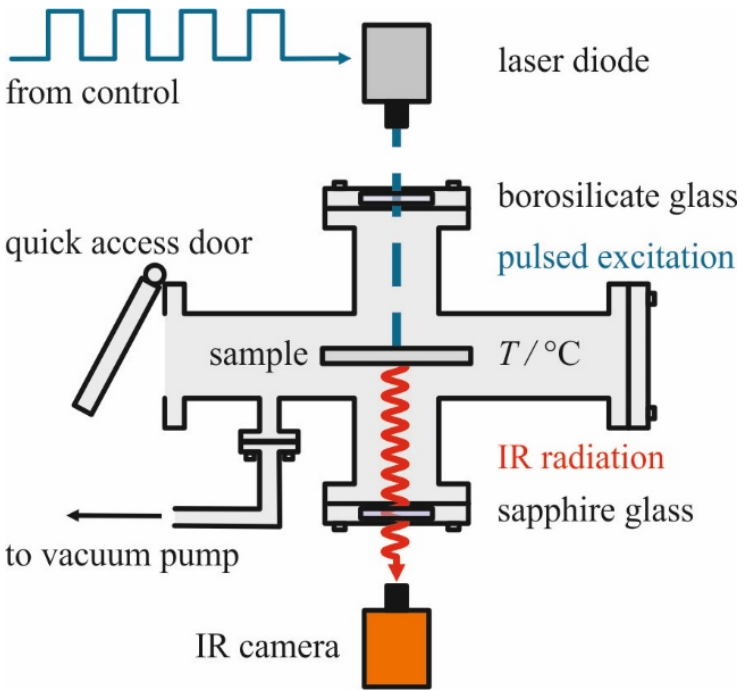

Fig. 1: Lock-in infrared thermography setup used for the thermal diffusivity measurements.
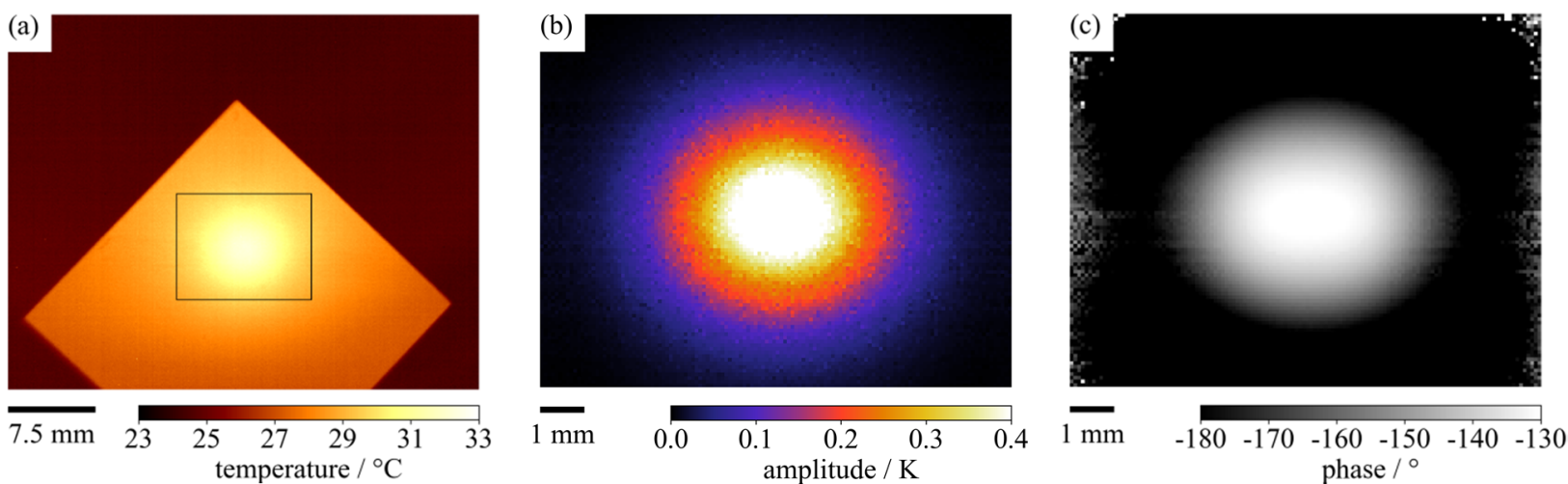

Fig. 2: Thermal image (a) of a $\mathrm{LiB}$ glass with a $\mathrm{Li}_{2} \mathrm{O}$ concentration of $14.29 \mathrm{~mol} \%$ under pulsed laser radiation at a lock-in frequency of $0.3 \mathrm{~Hz}$, together with close-up amplitude (b) and phase (c) images of the area indicated in (a).

The phase measurements are performed at a camera frequency of $198 \mathrm{~Hz}$ and over a measurement period of $480 \mathrm{~s}$. To ensure a high accuracy of the results, a range of lock-in frequencies is aimed for where the frequencydependent thermal diffusion length, $\mu$, of the material is less than half of the thickness of the sample. For $2 \mu \approx d$, the maximum error for a single data point amounts to $0.7 \%$ [10]. For the borate glass system, thermal diffusivity values in the order of $0.5 \cdot 10^{-6} \mathrm{~m}^{2} \mathrm{~s}^{-1}$ are expected. For a minimum lock-in frequency of $0.3 \mathrm{~Hz}$, this results in thermal diffusion lengths in the order of $0.73 \mathrm{~mm}$. The typical sample's thickness is between $1 \mathrm{~mm}$ and $1.5 \mathrm{~mm}$, i.e., the criterion $d>2 \mu$ is fulfilled for all samples and all lock-in frequencies used. At a camera frequency of $198 \mathrm{~Hz}$, a large number of images is taken within typical lock-in periods between $1 \mathrm{~s}(f=1 \mathrm{~Hz})$ and $3.3 \mathrm{~s}(f=0.3 \mathrm{~Hz})$. However, the maximum lock-in frequency is limited by the sensitivity of the camera, since the amplitude on the backside of the sample decreases exponentially with $d / \mu$.

\section{Results and Discussion}

Figure 2 shows a typical thermography image set used for the analysis. The amplitude (b) and phase images (c) are calculated from a series of temperature images (a). The amplitude and phase images show a pronounced maximum where the sample is hit by the laser beam. For the analysis of the thermal diffusivity, the maximum phase shift value is extracted from the phase image (c) at the region of the maximum of the corresponding amplitude image (b).

Series of measurements for undoped and terbium-doped LiB and LiAlB glasses are shown in figure 3. The measured phase values are normalized for the sample's thickness and plotted against the square root of the lock-in frequency. The slope corresponds to the fitting parameter, $b_{\text {norm }}$, which is used to calculate the thermal diffusivity values as shown in figure 4 and summarized in table 2. Sample sets with different $\mathrm{Li}_{2} \mathrm{O}$ concentration and $\mathrm{Tb}^{3+}$ content (with and without added $\mathrm{Al}_{2} \mathrm{O}_{3}$ as property modifier) are evaluated and their thermal diffusivity values added to figure 4 and table 2 . 

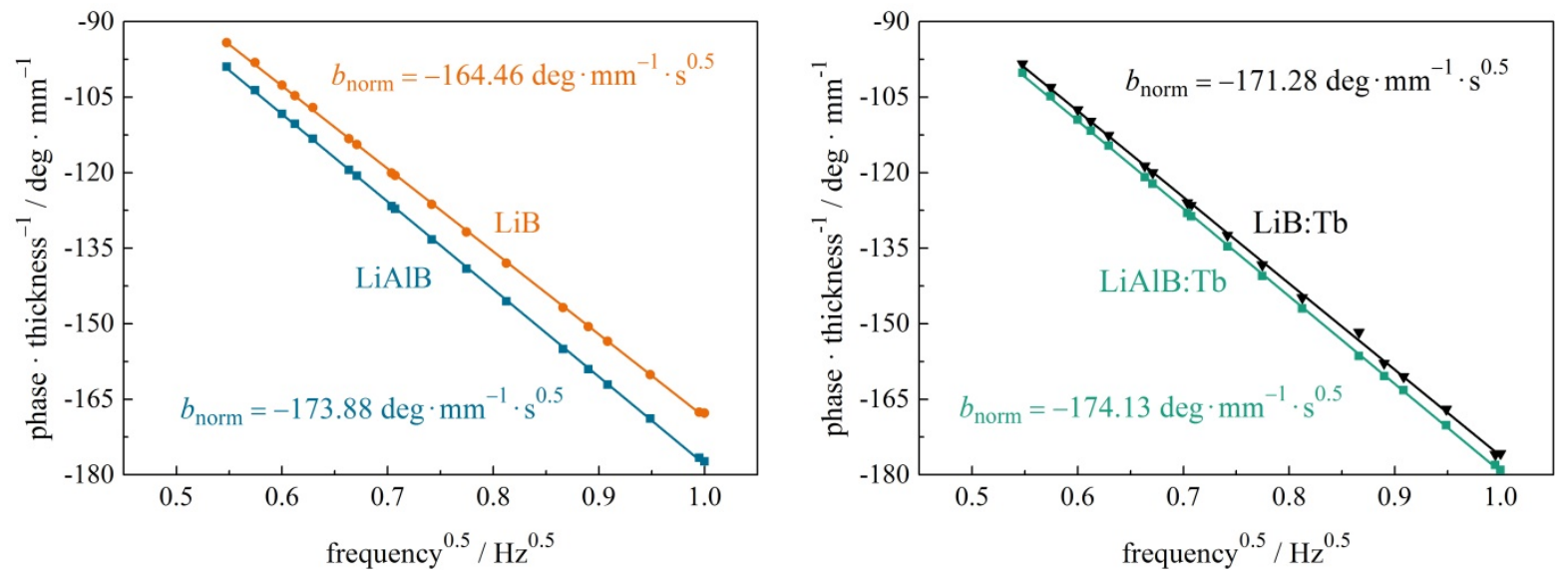

Fig. 3: Thickness normalized phase values for (left) undoped and (right) $T b^{3+}$ doped LiB and LiAlB glass. For the undoped glass, the $\mathrm{Li}_{2} \mathrm{O}$ concentration amounts to $14.29 \mathrm{~mol} \%$. For the terbium-doped samples, the $\mathrm{Li}_{2} \mathrm{O}$ concentration amounts to $25 \mathrm{~mol} \%$, while the $\mathrm{Tb}^{3+}$ content is 2.5 at. $\%$.
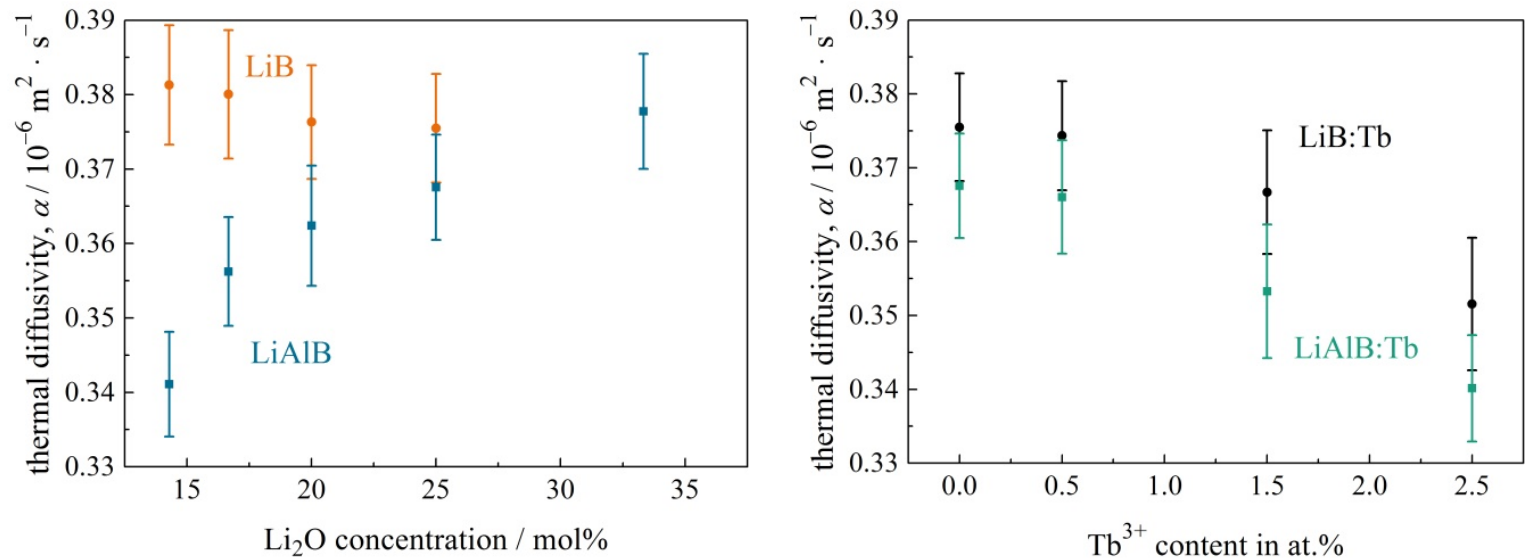

Fig. 4: Thermal diffusivity of (left) undoped and (right) $\mathrm{Tb}^{3+}$ doped LiB and LiAIB glass. For the terbium-doped samples, the $\mathrm{Li}_{2} \mathrm{O}$ concentration amounts to $25 \mathrm{~mol} \%$.

As it can be seen in figure 4, the chemical composition of the glass has a clear effect on the thermal diffusivity values, which change as the base glass network changes and because of additional doping with property modifiers or optical activators. In particular, the property modifier $\mathrm{Al}_{2} \mathrm{O}_{3}$ has a distinct effect on the thermal diffusivity of the glass compared to a glass with the same base component ratio but without additional property modifier (figure 4, left-hand side). The lower the lithium content, the more drastic is the decrease in thermal diffusivity caused by the added $\mathrm{Al}_{2} \mathrm{O}_{3}$. The glasses without $\mathrm{Al}_{2} \mathrm{O}_{3}$ only show a slight decrease in the thermal diffusivity with increasing lithium content. Additional terbium doping also leads to a slight decrease in the thermal diffusivity (up to $8 \%$ ). This applies for the $\mathrm{Al}_{2} \mathrm{O}_{3}$-free as well as the aluminum-containing sample set (figure 4 , right-hand side). 
Table 2. Thermal diffusivities of the various sample sets investigated.

\begin{tabular}{|c|c|c|c|c|}
\hline sample set & $\begin{array}{l}\mathrm{Li}_{2} \mathrm{O} \text { conc. } \\
/ \mathrm{mol} \%\end{array}$ & $\begin{array}{l}\mathrm{Tb}^{3+} \text { content } \\
/ \text { at. } \%\end{array}$ & $\begin{array}{l}\text { fitting parameter, } b_{\text {norm }} \\
I \mathrm{deg} \cdot \mathrm{mm}^{-1} \cdot \mathrm{s}^{0.5}\end{array}$ & $\begin{array}{l}\text { thermal diffusivity, } \alpha \\
/ 10^{-6} \mathrm{~m}^{2} \cdot \mathrm{s}^{-1}\end{array}$ \\
\hline LiB & $\begin{array}{l}14.29 \\
16.67 \\
20.00 \\
25.00\end{array}$ & $\begin{array}{l}- \\
- \\
- \\
-\end{array}$ & $\begin{array}{l}-164.46 \pm 0.52 \\
-164.73 \pm 0.30 \\
-165.55 \pm 0.27 \\
-165.73 \pm 0.33\end{array}$ & $\begin{array}{l}0.381 \pm 0.008 \\
0.380 \pm 0.009 \\
0.376 \pm 0.008 \\
0.375 \pm 0.007\end{array}$ \\
\hline LiAIB & $\begin{array}{l}14.29 \\
16.67 \\
20.00 \\
25.00 \\
33.33\end{array}$ & $\begin{array}{l}- \\
- \\
- \\
- \\
-\end{array}$ & $\begin{array}{l}-173.88 \pm 0.51 \\
-170.15 \pm 0.34 \\
-168.70 \pm 0.50 \\
-167.51 \pm 0.34 \\
-165.23 \pm 0.27\end{array}$ & $\begin{array}{l}0.341 \pm 0.007 \\
0.356 \pm 0.007 \\
0.362 \pm 0.008 \\
0.368 \pm 0.007 \\
0.378 \pm 0.008\end{array}$ \\
\hline $\mathrm{LiB}: \mathrm{Tb}$ & $\begin{array}{l}25.00 \\
25.00 \\
25.00\end{array}$ & $\begin{array}{l}2.5 \\
1.5 \\
0.5\end{array}$ & $\begin{array}{l}-171.28 \pm 0.97 \\
-167.70 \pm 0.32 \\
-165.98 \pm 0.22\end{array}$ & $\begin{array}{l}0.352 \pm 0.009 \\
0.367 \pm 0.008 \\
0.374 \pm 0.007\end{array}$ \\
\hline LiAIB:Tb & $\begin{array}{l}25.00 \\
25.00 \\
25.00\end{array}$ & $\begin{array}{l}2.5 \\
1.5 \\
0.5\end{array}$ & $\begin{array}{l}-174.13 \pm 0.52 \\
-170.86 \pm 0.89 \\
-167.86 \pm 0.49\end{array}$ & $\begin{array}{l}0.340 \pm 0.008 \\
0.353 \pm 0.009 \\
0.366 \pm 0.008\end{array}$ \\
\hline
\end{tabular}

\section{Conclusion}

Experiments on four different sets of borate glass samples showed that lock-in infrared thermography is a sensitive and suitable method to evaluate the thermal diffusivity of these glasses. The experimental phase values showed the expected dependency on the lock-in frequency. Changes in both the base glass composition and in doping levels are immediately detectable from the measured thermal diffusivity values. Assuming a reference value for thermal diffusivity is available or can be obtained for a known sample, the method can probably also be used to detect even small amounts of additives (wanted and unwanted) in these glasses. The accuracy of the measurements can be improved even further by using higher lock-in frequencies and more intense laser pulses.

\section{Acknowledgements}

The authors would like to thank the "Ministerium für Innovation, Wissenschaft und Forschung des Landes Nordrhein-Westfalen" for their financial support of the Fraunhofer Application Center for Inorganic Phosphors in Soest and within the FH STRUKTUR 2014 project "LED-2020". In addition, the authors would like to thank the German Federal Ministry for Education and Research (BMBF) for its support within the FHprofUnt 2014 project "LED-Glas" (project no. 03FH056PX4).

\section{REFERENCES}

[1] N. Soga, K. Hirao, M. Yoshimoto, and H. Yamamoto, J. Appl. Phys. 63, 4451 (1988).

[2] H. Lin, E. Y.-B. Pun, X. Wang, and X. Liu, J. Alloys Compd. 390, 197 (2005).

[3] M. Bengisu, J. Mater. Sci. 51, 2199 (2016).

[4] C. J. M. Lasance and A. Poppe, Thermal Management for LED Applications, 2014th ed. (Springer, New York, 2013).

[5] E. Zanoni, M. Meneghini, N. Trivellin, M. Dal Lago, and G. Meneghesso, in 2014 15th Int. Conf. Therm. Mech. Multi-Phys. Simul. Exp. Microelectron. Microsyst. Eurosime (2014), pp. 1-5.

[6] T. Q. Khanh, P. Bodrogi, T. Q. Vinh, and H. Winkler, LED Lighting: Technology and Perception (Wiley-VCH Verlag $\mathrm{GmbH} \&$ Co. KGaA, 2014).

[7] O. Breitenstein, Lock-in Thermography: Basics and Use for Evaluating Electronic Devices and Materials, (Springer, 2010).

[8] F. Steudel, S. Loos, B. Ahrens, and S. Schweizer, Phys. Scr. 90, 094004 (2015).

[9] F. Steudel, A. C. Rimbach, S. Loos, B. Ahrens, and S. Schweizer, Radiat. Meas. 90, 274 (2016).

[10] C. Boué and S. Holé, Infrared Phys. Technol. 55, 376 (2012). 